\title{
A Note on the Stability of a Modified Lotka-Volterra Model using Hurwitz Polynomials
}

\author{
FABIÁN TOLEDO SÁNCHEZ ${ }^{1}$, PEDRO PABLO CÁRDENAS ALZATE² AND CARLOS \\ ARTURO ESCUDERO SALCEDO ${ }^{3}$ \\ ${ }^{1,2}$ Department of Mathematics and GEDNOL \\ ${ }^{3}$ Department of Mathematics \\ ${ }_{1,2,3}$ Universidad Tecnológica de Pereira \\ Pereira, COLOMBIA
}

\begin{abstract}
In the analysis of the dynamics of the solutions of ordinary differential equations we can observe whether or not small variations or perturbations in the initial conditions produce small changes in the future; this intuitive idea of stability was formalized and studied by Lyapunov, who presented methods for the stable analysis of differential equations. For linear or nonlinear systems, we can also analyze the stability using criteria to obtain Hurwitz type polynomials, which provide conditions for the analysis of the dynamics of the system, studying the location of the roots of the associated characteristic polynomial. In this paper we present a stability study of a Lotka-Volterra type model which has been modified considering the carrying capacity or support in the prey and time delay in the predator, this stable analysis is performed using stability criteria to obtain Hurwitz-type polynomials.
\end{abstract}

Key-Words: Stability of differential equations, Hurwitz Polynomials, Hurwitz Criteria, Modified LotkaVolterra Model.

Received: March 7, 2021. Revised: July 20, 2021. Accepted: August 14, 2021. Published: September 7, 2021.

\section{Introduction}

The study of sensitive variations in the initial conditions is a fundamental work in the qualitative analysis of ordinary differential equations. This intuitive idea of stability for differential equations is the object of study in mathematical models applied to various disciplines of the exact sciences and control theory where the aim is to provide necessary and sufficient conditions so that, in a given problem with established initial conditions, solutions close to this initial value remain close throughout the future or in a given case tend to the equilibrium solution. The physicist Aleksandr Lyapunov proposed the basis for this concept of proximity in his doctoral thesis called The General Problem of Motion Stability [1], where he presented two methods to establish the stability of systems of ordinary differential equations

We can also establish the stability of a system of ordinary differential equations, either linear or nonlinear, using Hurwitz type polynomials. If the system is expressed in the form $\dot{\boldsymbol{x}}=\mathrm{Ax}$, then the problem of analyzing the stability becomes an algebraic problem, since it is enough to know the roots of the characteristic polynomial associated to the matrix $\boldsymbol{A}$, which correspond to the eigenvalues, and observe if they have a negative real part; if this is the case, the system $\dot{\boldsymbol{x}}=A \mathbf{x}$ is said to be asymptotically stable. If the polynomial has this characteristic it is said to be a Hurwitz polynomial [2], [3].

Therefore, to analyze the stability of a system of differential equations of the form $\dot{\boldsymbol{x}}=\mathrm{Ax}$ we can establish criteria to determine when the characteristic polynomial associated to the matrix $A$ is Hurwitz, that is, if all its roots have negative real part. There are several stability criteria to obtain Hurwitz type polynomials, these criteria present some equivalences in their formulation, among them are the Routh-Hurwitz criterion, the Lienard-Chipart conditions, the Hermite-Biehler theorem, the stability test and the Routh algorithm [4], [5], [6].

By way of introduction, in the study of the dynamics of ecological systems, food chains are of great importance to analyze the interaction between two or more species in relation to the dynamics between predators and their respective prey. These dynamics are generally modeled by extensions obtained from the classical Lotka-Volterra competition model [7]. Modifications of the Lotka-Volterra model seek to obtain a more realistic model when more factors are 
involved in an ecological system [8], for example, prey growth when affected by the Allee effect, monotonic or non-monotonic predator functional response described by Holling by a consumption rate function describing the amount of prey a predator can consume in a unit of time, logistic type growth function such as the Leslie-Gower model [9]-[14] . In this paper we consider the LotkaVolterra model with prey carrying capacity and predator time delay [15].

Indeed, the purpose of this paper is to analyze the stability of a Lotka-Volterra type model that has been modified by including carrying capacity or support in the prey and time delay in the predator, using the Routh-Hurwitz stability criteria and the Routh algorithm. The first section presents the Routh-Hurwitz stability criteria and Routh's algorithm; the second section initially presents a stability analysis of the classical Lotka-Volterra model and then presents the deduction and stability analysis of the modified model, using the criteria presented to obtain Hurwitz type polynomials and finally the results are analyzed through simulations performed in MATLAB for the solution of the system of nonlinear differential equations. With the simulations, the results obtained on the stability of the modified Lotka-Volterra model are validated.

\section{Hurwitz Polynomials}

Consider a system of linear or nonlinear differential equations expressed by,

$$
\dot{x}=A x,
$$

where $A$ is a square matrix and $\boldsymbol{x}$ is a vector. The stability of the system (1) at its equilibrium point can be determined by performing a study of the eigenvalues of the associated matrix $A$. This algebraic study establishes that if the roots of the characteristic polynomial associated to the matrix $A$ have negative real part, then the system (1) is asymptotically stable.

Definition 1: A polynomial with real coefficients is said to be Hurwitz if all its roots have negative real part, that is, if all its roots lie in $\mathbb{C}^{-}$, the left halfplane of the complex plane,

$$
\mathbb{C}^{-}=\{a+b i: a<0\} .
$$

The problem of determining the stability in a system of differential equations from the study of the roots of the characteristic polynomial associated with the linear or nonlinear system (1), translates into the task of finding necessary and sufficient conditions for which all the roots of this polynomial are located in the left half of the complex plane.

The question of obtaining Hurwitz type polynomials was initially proposed by the physicist and mathematician James Maxwell in 1868, who presented a solution to this problem for polynomials of degree 3, then in 1877, the Canadian mathematician Edward Routh presented an algorithm to solve the problem in a more general way providing explicit conditions for polynomials up to degree 5 and later it was the German mathematician Adolf Hurwitz in 1895 who formulated a properly analytical solution to the problem.

\subsection{Criteria for Obtaining Hurwitz Polynomials}

Let

$$
P(\lambda)=a_{0} \lambda^{n}+\cdots+a_{n-2} \lambda^{2}+a_{n-1} \lambda+a_{n},(3)
$$

be the characteristic polynomial associated to the matrix $A$ of the system (1).

Several mathematicians and physicists directed their studies in the search for algorithms to determine the location of the roots of the polynomial (3). A. Stodola at the end of the 19th century was interested in the problem of finding conditions under which all the roots of a polynomial have negative real part, but in 1895 it was Hurwitz who presented a solution of how to determine if the polynomial (3) has its roots in $\mathbb{C}^{-}$, based on the work of Hermite.

There are several criteria to obtain Hurwitz type polynomials. In this article we only briefly present the Routh-Hurwitz criterion and the Routh algorithm.

\subsubsection{Routh-Hurwitz criterion}

First we construct the following matrix called Hurwitz matrix, from the coefficients $a_{0}, a_{1}, \ldots, a_{n}$ of the polynomial (3):

$$
\mathcal{H}=\left[\begin{array}{cccccc}
a_{1} & a_{3} & a_{5} & a_{7} & \cdots & 0 \\
a_{0} & a_{2} & a_{4} & a_{6} & \cdots & 0 \\
0 & a_{1} & a_{3} & a_{5} & \cdots & 0 \\
0 & a_{0} & a_{2} & a_{4} & \cdots & 0 \\
\vdots & \vdots & \vdots & \vdots & \ddots & 0 \\
0 & 0 & 0 & 0 & \cdots & a_{n}
\end{array}\right] .
$$


This matrix has the following characteristics:

- The first row is formed by the coefficients of the polynomial (3) with odd location starting with $a_{1}$.

- The second row is formed by the coefficients of the polynomial (3) with even location starting with $a_{0}$.

- The elements of each subsequent row are formed so that the component $h_{i j}$ is given by:

$$
h_{i j}=\left\{\begin{array}{cr}
a_{2 j-i} & \text { si } 0<2 j-i \leq n \\
0 & \text { in other case }
\end{array}\right.
$$

In the Hurwitz matrix the coefficients $a_{1}, a_{2}, a_{3}$, $\ldots, a_{n}$ of the polynomial (3) are on the main diagonal, and all the elements of the last column are null, except the last element which is $a_{n}$.

Routh-Hurwitz theorem. The polynomial (3), with its positive leading coefficient $\left(a_{0}>0\right)$, is a Hurwitz polynomial if and only if all the diagonal principal minors of the Hurwitz Matrix $\mathcal{H}$ are positive [10].

Let us note that the diagonal principal minors of matrix (4) are given by the following determinants,

$$
\begin{gathered}
\Delta_{1}=\left|a_{1}\right|, \Delta_{2}=\left|\begin{array}{ll}
a_{1} & a_{3} \\
a_{0} & a_{2}
\end{array}\right|, \Delta_{3}=\left|\begin{array}{ccc}
a_{1} & a_{3} & a_{5} \\
a_{0} & a_{2} & a_{4} \\
0 & a_{1} & a_{3}
\end{array}\right|, \\
\Delta_{4}=\left|\begin{array}{cccc}
a_{1} & a_{3} & a_{5} & a_{7} \\
a_{0} & a_{2} & a_{4} & a_{6} \\
0 & a_{1} & a_{3} & a_{5} \\
0 & a_{0} & a_{2} & a_{4}
\end{array}\right|, \cdots, \Delta_{n}=a_{n} \cdot \Delta_{n-1} .
\end{gathered}
$$

For the particular case of a polynomial of degree three, we must verify only the conditions $a_{1}>0$ and $a_{1} a_{2}-a_{0} a_{3}>0$.

\subsubsection{Routh criterion}

The Routh arrangement for the coefficients of the polynomial (3) is given by,

$$
\begin{array}{ccccc}
a_{0} & a_{2} & a_{4} & a_{6} & \cdots \\
\overline{a_{1}} & a_{3} & a_{5} & a_{7} & \cdots \\
\hline b_{0} & b_{1} & b_{2} & b_{3} & \cdots \\
\hline c_{0} & c_{1} & c_{2} & c_{3} & \cdots \\
d_{0} & d_{1} & d_{2} & d_{3} & \cdots \\
\vdots & \vdots & \vdots & \vdots & \ddots
\end{array}
$$

This algorithm has been constructed taking into account the following steps:

- In the first row are the coefficients of the polynomial (3) with even location, starting with $a_{0}$.

- In the second row are the coefficients of the polynomial (3) with odd location, starting with $a_{1}$.

- The elements of each subsequent row are formed according to the following algorithm

$$
\begin{aligned}
& b_{0}=a_{2}-\frac{a_{0}}{a_{1}} a_{3}, \quad b_{1}=a_{4}-\frac{a_{2}}{a_{3}} a_{5}, \\
& c_{0}=a_{3}-\frac{a_{1}}{b_{0}} b_{1}, \quad c_{1}=a_{5}-\frac{a_{3}}{b_{1}} b_{2}, \quad \cdots \\
& d_{0}=b_{1}-\frac{b_{0}}{c_{0}} c_{1}, \quad d_{1}=b_{2}-\frac{b_{1}}{c_{1}} c_{2}, \quad \cdots
\end{aligned}
$$

\section{Routh's theorem:}

The number of roots of the polynomial $P(\lambda)$ in the right half-plane of the complex plane is equal to the number of sign variations of the first column in Routh's array (5).

\section{Routh criterion:}

The polynomial $P(\lambda)$ is Hurwitz polynomial if and only if when performing Routh's array (5) all values in the first column are nonzero of the same sign [11].

For the particular case of a polynomial of degree 3, the algorithm would be given by:

$$
\begin{array}{ll}
a_{0} & a_{2} \\
a_{1} & a_{3} \\
b_{0} &
\end{array}
$$

Therefore, according to Routh's criterion, a polynomial of degree 3 is Hurwitz if and only if $a_{0}>0, a_{1}>0$ and $b_{0}>0$.

\section{Lotka-Volterra Models}

In the 1920s, mathematicians Alfred Lotka and Vito Volterra simultaneously but independently proposed a nonlinear differential equation model to describe the population dynamics of two interacting species, a predator and its prey; they hoped to explain the increase of predators and, therefore, the decrease of 
prey in various ecological environments [16]. The model also called prey-predator system is given by:

$$
\left\{\begin{array}{l}
\dot{P}=\varepsilon P-\alpha P Q \\
\dot{Q}=-\gamma Q+\beta Q P
\end{array}\right.
$$

where, $P(t)$ and $Q(t)$ represent the prey and predator populations at time $t$ respectively; parameters $\varepsilon, \alpha, \gamma$ and $\beta$ are positive constants. $\varepsilon$ represents the natural growth rate of the prey in the absence of a predator, $\alpha$ represents the effect of the predator on the prey population, $\beta$ represents the effect of the prey on the predator population and $\gamma$ represents the natural mortality rate of the predator in the absence of a prey. It should be noted that since we are dealing with population dynamics, we only consider $P, Q>0$.

Our first job is to locate the equilibrium points which occur when $\dot{P}=0$ and $\dot{Q}=0$, that is, $\varepsilon P-$ $\alpha P Q=0$ and $-\gamma Q+\beta P Q=0$, which are located at the origin $\mathcal{O}=(0,0)$ and at $\mathcal{D}=\left(\frac{\gamma}{\beta}, \frac{\varepsilon}{\alpha}\right)$.

Let us analyze the stability at the equilibrium points $\mathcal{O}$ and $\mathcal{D}$. The Jacobian matrix of the system (6) is given by:

$$
\mathcal{J}(P, Q)=\left[\begin{array}{cc}
\varepsilon-\alpha Q & -\alpha P \\
\beta Q & -\gamma+\beta P
\end{array}\right] .
$$

Now, linearizing the flow in $\mathcal{O}$, we have that,

$$
\mathcal{J}(0,0)=\left[\begin{array}{cc}
\varepsilon & 0 \\
0 & -\gamma
\end{array}\right]
$$

whose characteristic polynomial is given by

$$
\mathcal{P}(\lambda)=\lambda^{2}+(\gamma-\varepsilon) \lambda-\varepsilon \gamma .
$$

Let us note that $\mathcal{P}(\lambda)$ is a Hurwitz polynomial if and only if all its coefficients are positive, that is, $a_{0}=$ 1, $a_{1}=\gamma-\varepsilon$ and $a_{2}=-\varepsilon \gamma$ are positive, this occurs when $\gamma>\varepsilon$ and $\varepsilon \gamma<0$, which we do not have since the parameters $\varepsilon$ and $\gamma$ are positive; therefore $\mathcal{P}(\lambda)$ is not Hurwitz and indeed the system (6) is not asymptotically stable in $\mathcal{O}$. We can interpret the above as follows, the equilibrium solution $P(t) \equiv 0$ and $Q(t) \equiv 0$ describe the simultaneous extinction of the populations of the prey $P$ and the predator $Q$, therefore, stable analysis at this equilibrium point is not of interest. Now let us analyze the stability at the equilibrium point $\mathcal{D}$, the linearized flow at this point is given by:

$$
\mathcal{J}\left(\frac{\gamma}{\beta}, \frac{\varepsilon}{\alpha}\right)=\left[\begin{array}{cc}
0 & \frac{-\alpha \gamma}{\beta} \\
\frac{\beta \varepsilon}{\alpha} & 0
\end{array}\right]
$$

whose characteristic polynomial is,

$$
\mathcal{P}(\lambda)=\lambda^{2}+\varepsilon \gamma
$$

Let us note that the polynomial $\mathcal{P}(\lambda)(7)$ is not Hurwitz as it has a null coefficient, and indeed the equilibrium point $\mathcal{D}$ is not asymptotically stable. The eigenvalues for this case are given by $\lambda=$ $\pm \sqrt{\varepsilon \gamma} i$ and since we have null real part, then we cannot establish asymptotic stability, however, point $\mathcal{D}$ is stable. We can make this determination by looking for a Lyapunov $\mathcal{L}$ function.

In fact, we are looking for a function of the form

$$
\mathcal{L}(P, Q)=U(P)+V(Q) .
$$

Now, calculating $\dot{\mathcal{L}}$ we have that

$$
\begin{array}{rlc}
\dot{\mathcal{L}}(P, Q) & =\frac{d U}{d P} \dot{P}+\frac{d U}{d Q} \dot{Q} \\
& =\frac{d U}{d P}(\varepsilon P-\alpha P Q)+\frac{d U}{d Q}(-\gamma Q+\beta P Q) .
\end{array}
$$

Making $\dot{\mathcal{L}}(P, Q)=0$ and integrating we have that,

$$
\begin{aligned}
& U(P)=\alpha P-\varepsilon \ln P, \\
& V(P)=\beta Q-\gamma \ln Q .
\end{aligned}
$$

Therefore, we have

$$
\mathcal{L}(P, Q)=\alpha P-\varepsilon \ln P+\beta Q-\gamma \ln Q .
$$

Let us note that $\mathcal{L}(P, Q)$ is positive definite, i.e., $\mathcal{L}(P, Q)>0$ for all $P, Q>0$ and $(P, Q) \neq\left(\frac{\gamma}{\beta}, \frac{\varepsilon}{\alpha}\right)$, moreover, $\mathcal{L}\left(\frac{\gamma}{\beta}, \frac{\varepsilon}{\alpha}\right)=0$. Since also $\dot{\mathcal{L}}(P, Q) \leq 0$, then the equilibrium point $\mathcal{D}$ is stable in the Lyapunov sense. The equilibrium solutions corresponding to $P(t)=\frac{\gamma}{\beta}$ and $Q(t)=\frac{\varepsilon}{\alpha}$ describe the only non-zero constants so that the populations of prey $P$ and predator $Q$ can permanently coexist. Figure 1. presents the plots of $P(t)$ and $Q(t)$ where the interaction of prey and predator is observed and the Figures 2. and 3. shows the field of slopes and the trajectories near the equilibrium point $\mathcal{D}$ for $\varepsilon=$ $\alpha=\gamma=\beta=1$. 


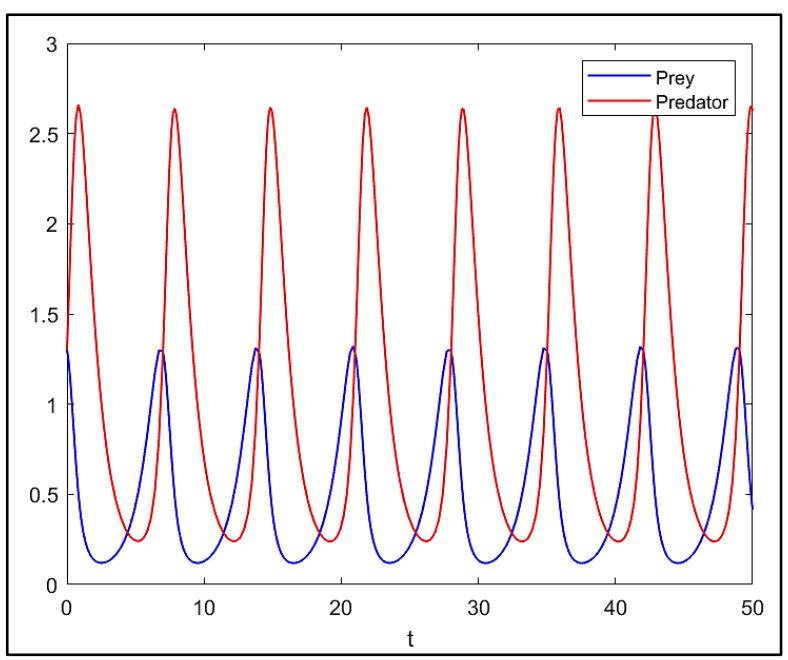

Fig.1: Graphs of $P(t)$ and $Q(t)$, with initial condition $(1.2,1.2)$.

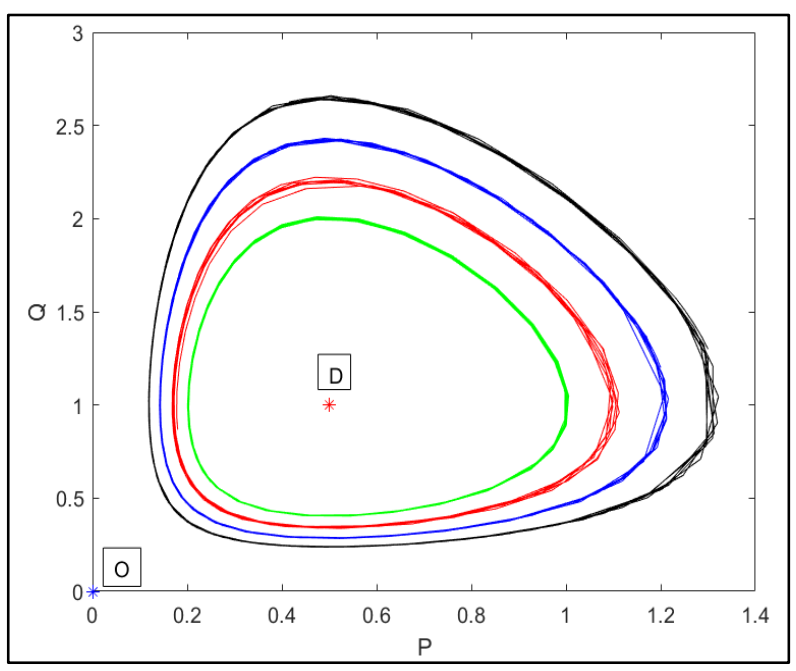

Fig.2: Trajectories for the system (6). The equilibrium point $\mathrm{D}$ is stable.

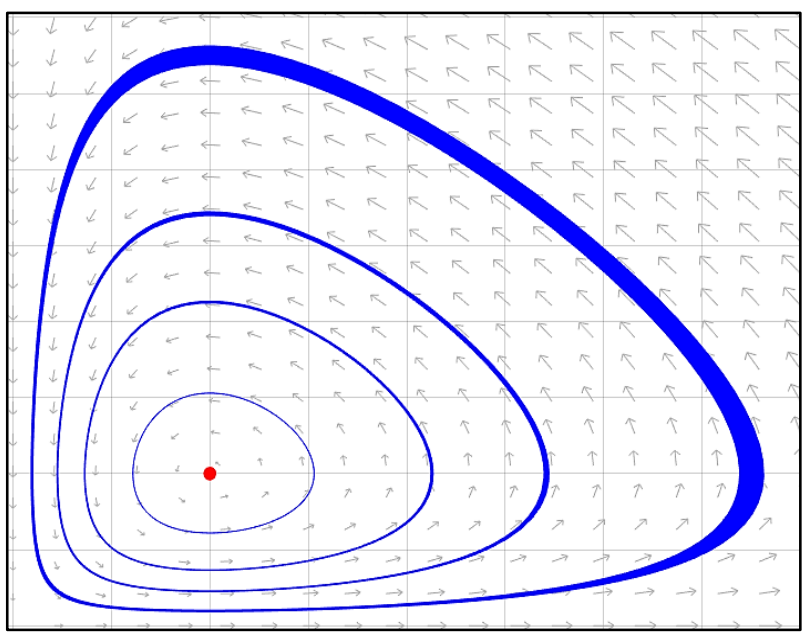

Fig.3: Field of slopes and trajectories for the system (6).
In Figure 1. the graphs of the prey $P$ and the predator $Q$ are shown with respect to time, where it is observed that they remain orbiting as time passes and in the Figures 2. and 3. show the trajectories of the Lotka-Volterra system (6) where two species interact, orbiting around their stable equilibrium point.

\subsection{Modified Lotka-Volterra Model}

In this section we will present modifications of the classical Lotka-Volterra model (6), which is improved in the sense that it takes into account the intraspecific competition of the prey and the current growth rate of the predator, which depends not only on the present amount of food but also on past amounts, for example, in a gestation period, therefore, a lag term is incorporated in the differential equation relative to the predator.

Initially, let us consider the Lotka-Volterra model with bearing capacity $K>0$ in the dams, given by:

$$
\left\{\begin{array}{c}
\dot{P}=\varepsilon P\left(1-\frac{P}{K}\right)-\alpha P Q, \\
\dot{Q}=-\gamma Q+\beta Q P .
\end{array}\right.
$$

Where the prey specific growth rate, predation rate, predator mortality rate and conversion rate $\varepsilon, \alpha, \gamma$ and $\beta$, are given as in (6). Now, if we take into account in the predator population $Q$, the prey density in the past, we have that the system (7) becomes:

$$
\left\{\begin{array}{lcc}
\dot{P} & = & \varepsilon P\left(1-\frac{P}{K}\right)-\alpha P Q, \\
\dot{Q} & = & -\gamma Q+\beta Q \int_{-\infty}^{t} P(s) D(t-s) d s,
\end{array}\right.
$$

where

$$
D(t)=a e^{-a t},
$$

with $a>0$, is a type of weight function.

Let us note that if $a$ is small, the larger the time interval in the past in which the values of the dam $P$ are taken into account.

If

$$
R(t)=\int_{-\infty}^{t} P(s) D(t-s) d s,
$$

then we can transform the system (8) into the following system of three differential equations: 


$$
\left\{\begin{array}{llc}
\dot{P} & = & \varepsilon P\left(1-\frac{P}{K}\right)-\alpha P Q \\
\dot{Q} & = & -\gamma Q+\beta Q R \\
\dot{R} & = & a(P-R)
\end{array}\right.
$$

If $P=K x_{1}, Q=k x_{2}, R=K x_{3}$ and $t=\frac{\tau}{\varepsilon}$, then the system (9) becomes,

$$
\left\{\begin{array}{lll}
\dot{x_{1}} & = & x_{1}\left(1-x_{1}\right)-\frac{\alpha K}{\varepsilon} x_{1} x_{2}, \\
\dot{x_{2}} & = & -\frac{\gamma}{\varepsilon} x_{2}+\frac{\beta K}{\varepsilon} x_{2} x_{3}, \\
\dot{x_{3}} & = & \frac{a}{\varepsilon}\left(x_{1}-x_{3}\right) .
\end{array}\right.
$$

To perform a stability analysis of the system (10) we start by establishing its equilibrium points, i.e., the $\tilde{x}$ such that $\mathrm{f}(\tilde{x})=0$. That is:

$$
\begin{array}{cl}
x_{1}\left(1-x_{1}\right)-\frac{\alpha K}{\varepsilon} x_{1} x_{2} & =0, \\
-\frac{\gamma}{\varepsilon} x_{2}+\frac{\beta K}{\varepsilon} x_{2} x_{3} & =0, \\
\frac{a}{\varepsilon}\left(x_{1}-x_{3}\right) & =0 .
\end{array}
$$

From (13) we have that $x_{1}=x_{3}$, and in effect equation (12) transforms into equation

$$
x_{2}\left(\frac{\beta K}{\varepsilon} x_{1}-\frac{\gamma}{\varepsilon}\right)=0
$$

And we have that $x_{2}=0$ or $\frac{\beta K}{\varepsilon} x_{1}-\frac{\gamma}{\varepsilon}=0$ and from equation (11) we have the equilibrium points.

These equilibrium points are given at the origin $\mathcal{O}=$ $(0,0,0)$ for all values of $\varepsilon, \alpha, \gamma, \beta, a$ and $K$, at the point $\mathcal{A}_{1}=(1,0,1)$ and $\mathcal{A}_{2}=\left(\frac{\gamma}{K \beta}, \frac{\varepsilon(K \beta-\gamma)}{\alpha \beta K^{2}}, \frac{\gamma}{K \beta}\right)$. Stability at the origin is not of interest, as mentioned for the Lotka-Volterra model (6). The Jacobian matrix of the system (10) is given by:

$$
\begin{gathered}
\mathcal{J}\left(x_{1}, x_{2}, x_{3}\right)= \\
{\left[\begin{array}{ccc}
1-2 x_{1}-\frac{\alpha K}{\varepsilon} x_{2} & -\frac{\alpha K}{\varepsilon} x_{1} & 0 \\
0 & \frac{-\gamma+K \beta x_{3}}{\varepsilon} & \frac{K \beta}{\varepsilon} x_{2} \\
\frac{a}{\varepsilon} & 0 & -\frac{a}{\varepsilon}
\end{array}\right] .}
\end{gathered}
$$

\subsubsection{Stability in $\mathcal{A}_{1}$}

The stability at the equilibrium point $\mathcal{A}_{1}=(1,0,1)$ is obtained by linearizing the flux at $\mathcal{A}_{1}$, i.e:

$$
\mathcal{J}(1,0,1)=\left[\begin{array}{ccc}
-1 & -\frac{\alpha K}{\varepsilon} & 0 \\
0 & \frac{-\gamma+K \beta}{\varepsilon} & 0 \\
\frac{a}{\varepsilon} & 0 & -\frac{a}{\varepsilon}
\end{array}\right]
$$

The characteristic polynomial can be expressed as

$$
\begin{gathered}
\mathcal{P}(\lambda)=\lambda^{3}+\left(\frac{a}{\varepsilon}+\frac{\gamma-K \beta}{\varepsilon}+1\right) \lambda^{2}+ \\
{\left[\frac{a}{\varepsilon}\left(\frac{\gamma-K \beta}{\varepsilon}+1\right)+\frac{\gamma-K \beta}{\varepsilon}\right] \lambda+\frac{a}{\varepsilon}\left(\frac{\gamma-K \beta}{\varepsilon}\right) .}
\end{gathered}
$$

To analyze the nature of the eigenvalues of the polynomial (14) that will determine the stability in $\mathcal{A}_{1}$, we will use the Hurwitz criterion.

Thus, the Hurwitz matrix associated with the polynomial $\mathcal{P}(\lambda)(14)$ for $a_{0}=1, a_{1}=\frac{a}{\varepsilon}+$ $\frac{\gamma-K \beta}{\varepsilon}+1, \quad a_{2}=\frac{a}{\varepsilon}\left(\frac{\gamma-K \beta}{\varepsilon}+1\right)+\frac{\gamma-K \beta}{\varepsilon}$ and $a_{3}=$ $\frac{a}{\varepsilon}\left(\frac{\gamma-K \beta}{\varepsilon}\right)$, is given by:

$\mathcal{H}=\left[\begin{array}{cc}\frac{a}{\varepsilon}+\frac{\gamma-K \beta}{\varepsilon}+1 & \frac{a}{\varepsilon}\left(\frac{\gamma-K \beta}{\varepsilon}\right) \\ 1 & \frac{a}{\varepsilon}\left(\frac{\gamma-K \beta}{\varepsilon}+1\right)+\frac{\gamma-K \beta}{\varepsilon}\end{array}\right]$.

Applying Hurwitz's theorem, we have that the polynomial $\mathcal{P}(\lambda)(14)$ is Hurwitz, if it is satisfied that:

$$
\begin{aligned}
\Delta_{1} & =\left|\beta \frac{a}{\varepsilon}+\frac{\gamma-K \beta}{\varepsilon}+1\right|>0, \\
\Delta_{2} & =\left|\begin{array}{cc}
\frac{a}{\varepsilon}+\frac{\gamma-K \beta}{\varepsilon}+1 & \frac{a}{\varepsilon}\left(\frac{\gamma-K \beta}{\varepsilon}\right) \\
1 & \frac{a}{\varepsilon}\left(\frac{\gamma-K \beta}{\varepsilon}+1\right)+\frac{\gamma-K \beta}{\varepsilon}
\end{array}\right| \\
>0, &
\end{aligned}
$$

$\Delta_{1}$ is positive if $\gamma>K \beta$ or what is the same if $\frac{\gamma}{K \beta}>$ 1 , because the other parameters $\beta$ and $\varepsilon$ are positive, we only need $\Delta_{2}$ to be positive, that is: 


$$
\begin{gathered}
{\left[\frac{a}{\varepsilon}+\frac{\gamma-K \beta}{\varepsilon}+1\right]\left[\frac{a}{\varepsilon}\left(\frac{\gamma-K \beta}{\varepsilon}+1\right)+\frac{\gamma-K \beta}{\varepsilon}\right]} \\
-\frac{a}{\varepsilon}\left(\frac{\gamma-K \beta}{\varepsilon}\right)>0 .
\end{gathered}
$$

Which is held for,

$$
\begin{aligned}
\frac{1}{\varepsilon}\left(\frac{\gamma-K \beta}{\varepsilon}+1\right)\left[\frac{a^{2}}{\varepsilon}+a\left(\frac{\gamma-K \beta}{\varepsilon}+1\right)+(\gamma\right. \\
-K \beta)]>0,
\end{aligned}
$$

which is also true for all $\frac{\gamma}{K \beta}>1$.

Therefore, the polynomial $\mathrm{P}(\lambda)$ (14) is Hurwitz if and only if $\frac{\gamma}{K \beta}>1$, and indeed the system (10) is asymptotically stable in $\mathcal{A}_{1}$ si $\frac{\gamma}{K \beta}>1$.

\subsubsection{Stability in $\mathcal{A}_{2}$}

The stability at the equilibrium point $\mathcal{A}_{2}=$ $\left(\frac{\gamma}{K \beta}, \frac{\varepsilon(K \beta-\gamma)}{\alpha \beta K^{2}}, \frac{\gamma}{K \beta}\right)$ is obtained by linearizing the flux at $\mathcal{A}_{2}$, i.e:

$$
\mathcal{J}\left(\mathcal{A}_{2}\right)=\left[\begin{array}{ccc}
-\frac{\gamma}{K \beta} & -\frac{\alpha \gamma}{\beta \varepsilon} & 0 \\
0 & 0 & \frac{\beta}{\alpha}\left(1-\frac{\gamma}{K \beta}\right) \\
\frac{a}{\varepsilon} & 0 & -\frac{a}{\varepsilon}
\end{array}\right] .
$$

The characteristic polynomial can be expressed as:

$$
\begin{gathered}
\mathcal{P}(\lambda)=\lambda^{3}+\left(\frac{a}{\varepsilon}+\frac{\gamma}{K \beta}\right) \lambda^{2}+\frac{a}{\varepsilon}\left(\frac{\gamma}{K \beta}\right) \lambda \\
+\frac{a \gamma}{\varepsilon K \beta}\left(\frac{K \beta-\gamma}{\varepsilon}\right)
\end{gathered}
$$

As in the previous case, to analyze the nature of the eigenvalues of the polynomial (15) that will determine the stability in $\mathcal{A}_{2}$ we use Routh's algorithm for $a_{0}=1, a_{1}=\frac{a}{\varepsilon}+\frac{\gamma}{K \beta}, a_{2}=\frac{a}{\varepsilon}\left(\frac{\gamma}{K \beta}\right)$ and $a_{3}=\frac{a \gamma}{\varepsilon K \beta}\left(\frac{K \beta-\gamma}{\varepsilon}\right)$.
First let's find the coefficient $b_{0}$, that is,

$$
b_{0}=\frac{a}{\varepsilon}\left(\frac{\gamma}{K \beta}\right)-\frac{1}{\frac{a}{\varepsilon}+\frac{\gamma}{K \beta}} \cdot \frac{a \gamma}{\varepsilon K \beta}\left(\frac{K \beta-\gamma}{\varepsilon}\right) .
$$

Thus, we have Routh's arrangement (5) given by

$$
\begin{array}{cc}
1 & \frac{a}{\varepsilon}\left(\frac{\gamma}{K \beta}\right) \\
\frac{a}{\varepsilon}+\frac{\gamma}{K \beta} & 2 \beta \sigma(\gamma-1) \\
b_{0} &
\end{array}
$$

Then, to apply Routh's theorem we have to analyze the signs of the first column of the algorithm (16), as $a_{0}$ and $a_{1}$ are positive, then we only have to analyze the sign of $b_{0}$, if $b_{0}$ is positive then the polynomial (15) is Hurwitz and in effect the equilibrium point $\mathcal{A}_{2}$ would be asymptotically stable. Now, we can see when $b_{0}$ is positive, that is,

$$
\frac{a}{\varepsilon}\left(\frac{\gamma}{K \beta}\right)-\frac{1}{\frac{a}{\varepsilon}+\frac{\gamma}{K \beta}} \cdot \frac{a \gamma}{\varepsilon K \beta}\left(\frac{K \beta-\gamma}{\varepsilon}\right)>0 .
$$

This is the case if

$$
\frac{\gamma}{K \beta}>\frac{\gamma(K \beta-\gamma)}{a K \beta+\varepsilon \gamma}
$$

or

$$
a>K \beta-\gamma-\frac{\varepsilon \gamma}{K \beta}
$$

Let $\tilde{a}=K \beta-\gamma-\frac{\varepsilon \gamma}{K \beta}$. Then we conclude that $b_{0}$ is positive when it is satisfied that $a>\tilde{a}$ and indeed by Routh's theorem as in the first column of the algorithm (16) the values of $a_{0}, a_{1}$ and $b_{0}$ are positive for the above constraint, it follows that the polynomial (15) is Hurwitz and therefore the equilibrium point $\mathcal{A}_{2}$ is asymptotically stable.

\section{Results and Discussion}

In this section we will analyze the asymptotic stability results obtained previously together with their respective simulation performed in MATLAB for the solution of the system of nonlinear differential equations. 


\subsection{Stability in $\mathcal{A}_{1}$}

If $\frac{\gamma}{K \beta}>1$ the equilibrium point $\mathcal{A}_{1}=(1,0,1)$ is asymptotically stable. This condition means that the limiting value of the prey density $K$ and the rate of prey conversion to predators $\beta$ are not large enough to sustain the predator population that eventually goes extinct, as seen in Figure 4.

Figure 5. presents the simulation for the case where $\frac{\gamma}{K \beta}>1$ for the particular cases where $\gamma=2$ and the other parameters equal to 1 , where indeed the equilibrium point $\mathcal{A}_{1}$ is asymptotically stable. It is important to see that in this figure four trajectories are presented for very close initial conditions.

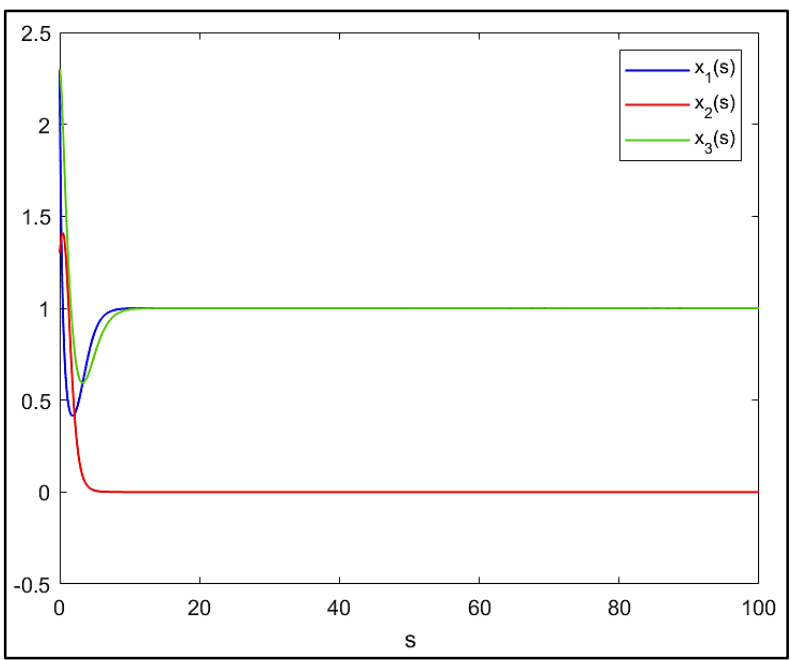

Fig.4: Graphs of $x_{1}, x_{2}$ and $x_{3}$, with initial condition $(1.4,2.4,2.4) . \mathcal{A}_{1}$ stable.

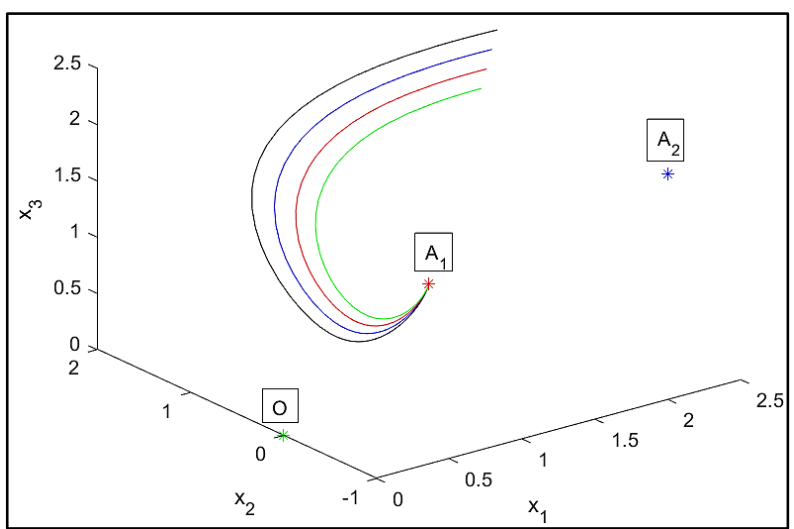

Fig.5: Trajectories of (10), with initial condition $(1.4,2.4,2.4),(1.5,2.5,2.5),(1.6,2.6,2.6)$ and $(1.7,2.7,2.7) . \mathcal{A}_{1}$ stable.

If $\frac{\gamma}{K \beta}<1$ the equilibrium point $\mathcal{A}_{1}=(1,0,1)$ is unstable, as seen in Figure 7. for $\beta=2$ and the value of the other parameters equal to 1 . For this case both prey and predators tend to extinction, as seen in Figure 6.

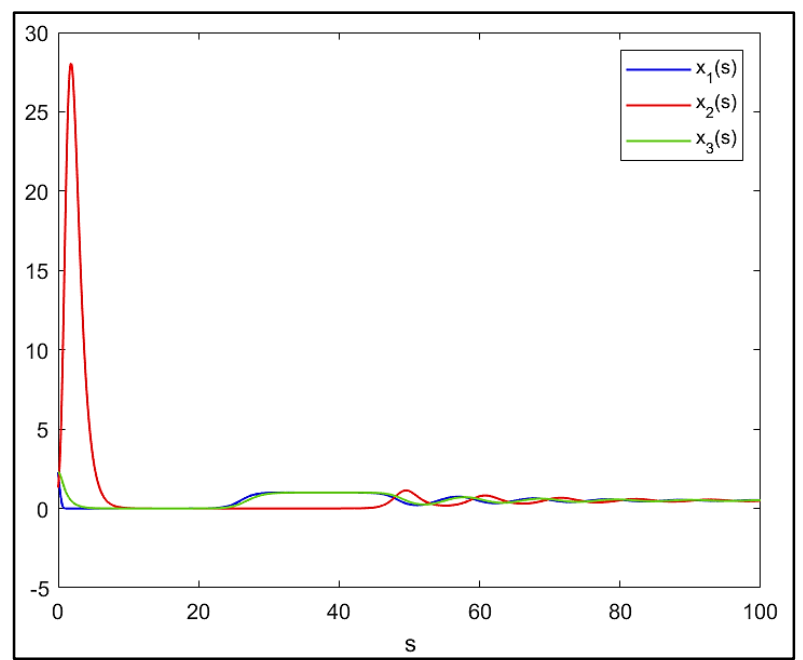

Fig.6: Graphs of $x_{1}, x_{2}$ and $x_{3} . \mathcal{A}_{1}$ unstable.

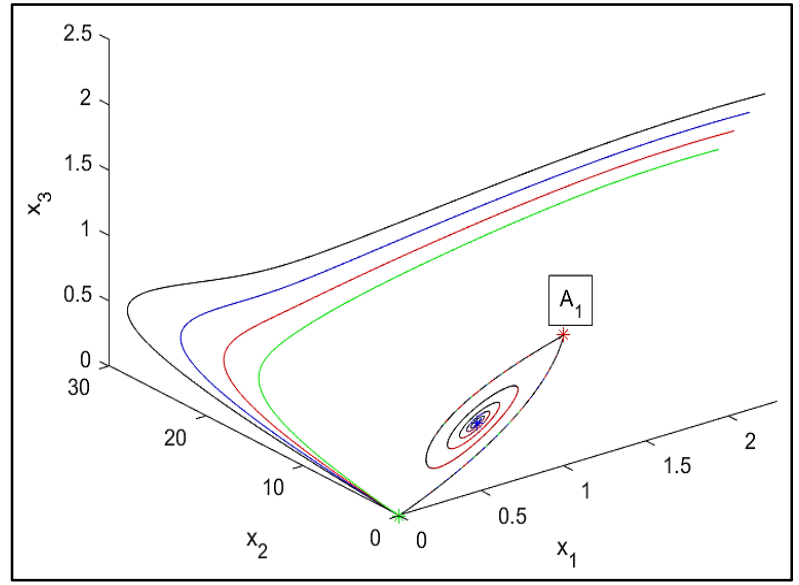

Fig.7: Trajectories of (10), with initial condition $(2,20,1.5),(2.1,21,1.6),(2.2,22,1.7) \quad$ and $(2.3,23,1.8) . \mathcal{A}_{1}$ unstable.

\subsection{Stability in $\mathcal{A}_{2}$}

To establish stability at the equilibrium point $\mathcal{A}_{2}=$ $\left(\frac{\gamma}{K \beta}, \frac{\varepsilon(K \beta-\gamma)}{\alpha \beta K^{2}}, \frac{\gamma}{K \beta}\right)$ we must first take into account that $\mathcal{A}_{2}$ must be positive for the model to make ecological sense, this is when $\mathcal{A}_{2}$ is in the positive octant in the space $x_{1}-x_{2}-x_{3}$, that is, when $\gamma<$ $K \beta$ or what is the same if $\frac{\gamma}{K \beta}<1$.

According to the above, we can analyze the stability at the equilibrium point $\mathcal{A}_{2}$ if the following conditions are taken into account:
1) $\frac{\gamma}{K \beta}<1$.
2) $a>K \beta-\gamma-\frac{\varepsilon \gamma}{K \beta}$. 
In addition to these two conditions, we must analyze the sign of $\tilde{a}=K \beta-\gamma-\frac{\varepsilon \gamma}{K \beta}$, if $\tilde{a} \leq 0$, then the equilibrium point $\mathcal{A}_{2}$ is asymptotically stable. The value of the parameter a is of vital importance because it corresponds to the value that varies me the weight function $D(t)$, recall that if $a$ is small, the greater the time interval in the past in which the values of the dam $P$.

As a particular case, if condition 1) is satisfied for $\gamma=1, K=1$ and $\beta=2$ and we take a $\varepsilon=2$, then $\tilde{a}=0$ and indeed the equilibrium point $\mathcal{A}_{2}$ is asymptotically stable for all values of " $a>0$ ", as observed in Figure 8 and 9, but for if $a$ is small number we are losing stability at point $\mathcal{A}_{2}$, as observed in Figure 10 and 11.

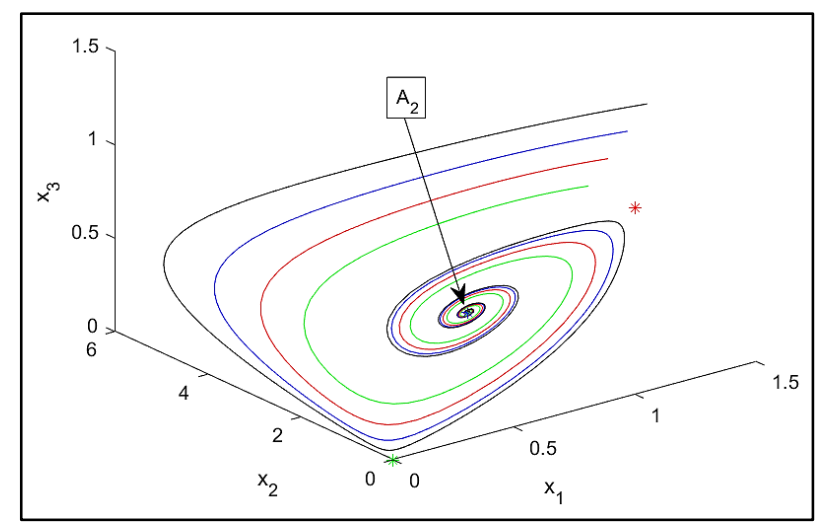

Fig.8: Trajectories of (10). $\mathcal{A}_{2}$ stable. $a=1$.

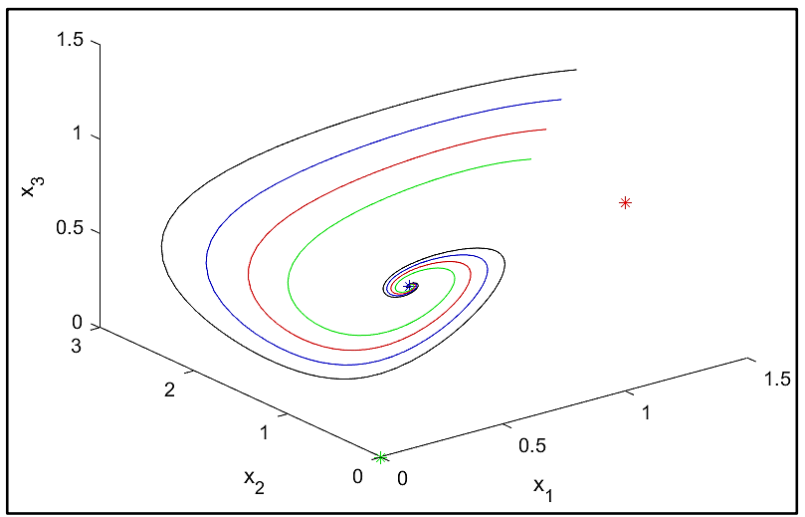

Fig.9: Trajectories of (10). $\mathcal{A}_{2}$ stable. $a=3$.

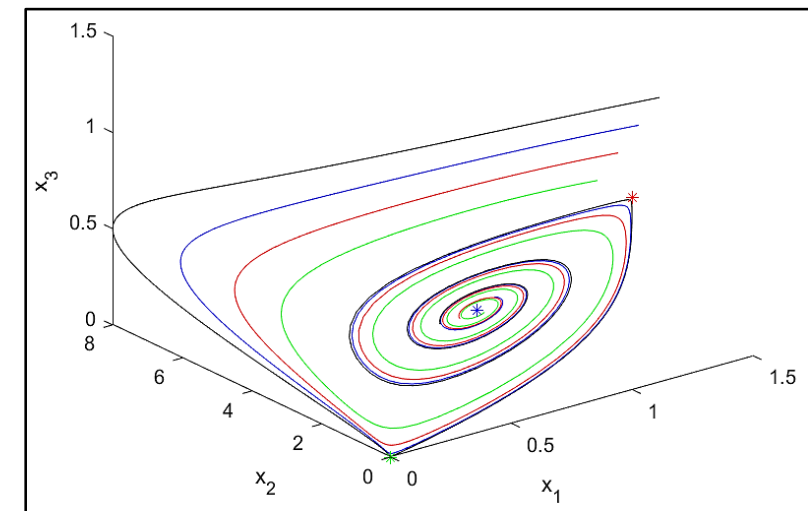

Fig.10: Trajectories of (10). $\mathcal{A}_{2}$ losing stability.

$$
a=0.6 \text {. }
$$

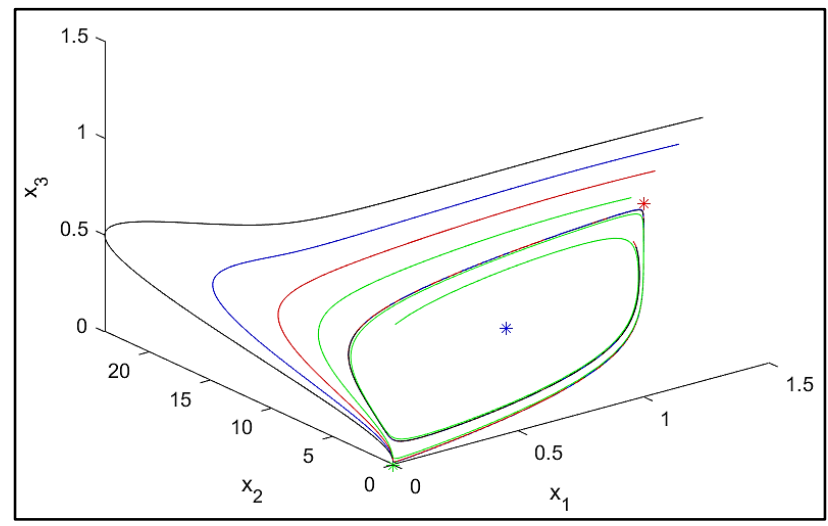

Fig.11: Trajectories of (10). $\mathcal{A}_{2}$ losing stability.

$$
a=0.3 \text {. }
$$

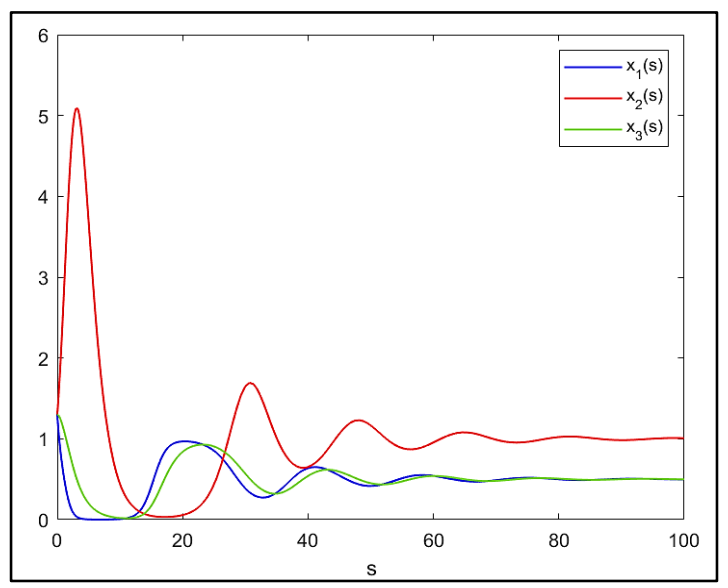

Fig.12: Graphs of $x_{1}, x_{2}$ and $x_{3} . \mathcal{A}_{2}$ stable. 


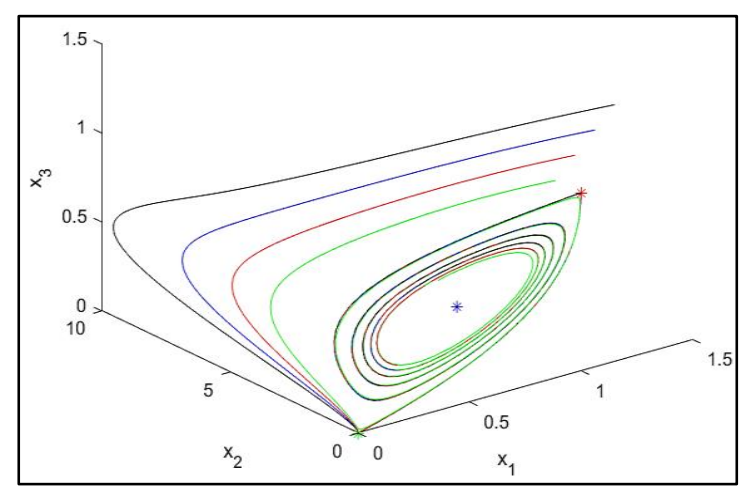

Fig.13: Trajectories of (10). $\mathcal{A}_{2}$ unstable.

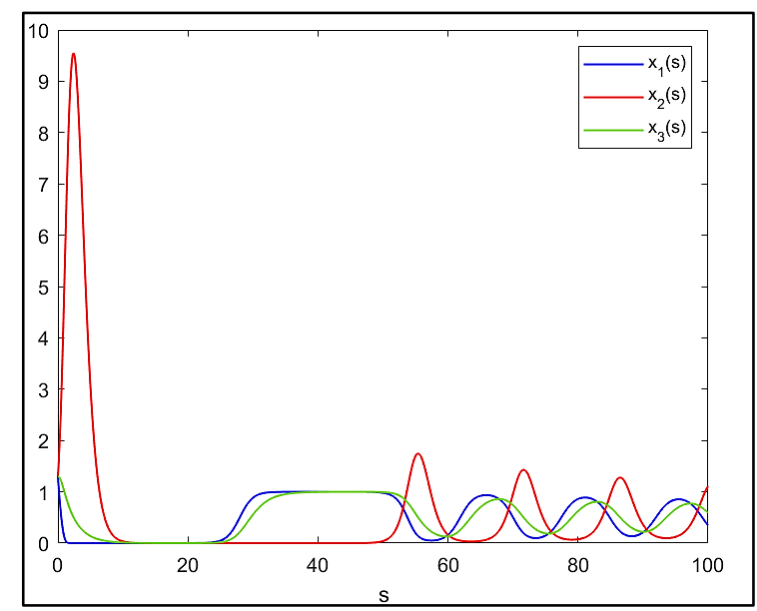

Fig.14: Graphs of $x_{1}, x_{2}$ and $x_{3} . \mathcal{A}_{2}$ unstable.

Now if condition 1) is satisfied for $\gamma=1, K=1$ and $\beta=2$ and we take a $\varepsilon=0.5$, then $\tilde{a}=0.75$ and indeed the equilibrium point $\mathcal{A}_{2}$ is unstable for all values of $a>0.75$, as observed in Figure 13. In Figures 12 and 14 we observe the plots of $x_{1}$ and $x_{2}$ corresponding to the prey and predators for the case where the equilibrium point $\mathcal{A}_{2}$ is stable and unstable respectively.

\section{Conclusion}

In the stability analysis of systems of differential equations, whether linear or nonlinear, the use of stability criteria to obtain Hurwitz type polynomials establishes necessary and sufficient conditions for the study of the dynamics of mathematical models. These algebraic stability criteria have made it possible to determine values for which the modified Lotka-Volterra model is asymptotically stable, as could be visualized by means of the simulations carried out in MATLAB, for the values provided by the Routh-Hurwitz and Routh criteria.

Regarding the dynamics of the modified LotkaVolterra model, the influence of the delay in the predator population provided by the condition $\tilde{a}$ obtained using the Routh criterion is observed. A relevant fact is about the dynamics of the system when $\tilde{a}>0$ which represents a bifurcation study.

The stability analysis of the modified LotkaVolterra model using Hurwitz type polynomials provides conditions that are necessary and sufficient to establish regions of stability for various values of the parameters involved in the model.

\section{Acknowledgements:}

The authors gratefully acknowledge the support of the Universidad Tecnológica de Pereira - Colombia, and the Group GEDNOL.

\section{References:}

[1] A. . Lyapunov, "The General Problem of the Stability of motion," vol. 6, no. 11, pp. 951952, 1992.

[2] B. Aguirre, C. Loredo, E. Díaz, and E. Campos, "Stability Systems Via Hurwitz Polynomials," vol. 24, no. 1, pp. 61-77, 2017.

[3] F. Toledo Sánchez, “Análsis de estabilidad de sistemas de ecuaciones difenciales utilizando polinomios de Hurwitz," Universidad Tecnologica de Pereira, 2020.

[4] B. Aguirre-Hernandez, C. A. LoderoVillalobos, and F. R. Garcia-Sosa, "a Proposal of Problems About Stable Hurwitz Polynomials," Cybern. Phys., vol. 2, no. 3, pp. 159-164, 2013.

[5] C. Lodero, "Factorización de Hadamard para polinomios Hurwitz," Universidad Autonoma Metropolitana, 2012.

[6] C. A. Loredo, "Criterios para determinar si un polinomio es polinomio Hurwitz," Universidad Autónoma Metropolitana Unidad Iztapalapa, 2005.

[7] M. Farkas, Periodic motions, Applied Ma. Springer-Verlag, 2018.

[8] T. N. Mishra and B. Tiwari, "Stability and Bifurcation Analysis of a Prey-Predator Model," Int. J. Bifurc. Chaos, vol. 31, no. 4, 2021.

[9] B. Xie and F. Xu, "Stability analysis for a time-delayed nonlinear predator-prey model," Adv. Differ. Equations, vol. 2018, no. 1, pp. 1-16, 2018.

[10] F. Javier and R. Bahamón, "On the dynamics of Leslie-Gower type predator-prey model with non-monotonic functional response and Allee effect on prey," 2017. 
[11] J. Zhang, L. Zhang, and Y. Bai, "Stability and bifurcation analysis on a predator-prey system with the weak Allee effect," Mathematics, vol. 7, no. 5, pp. 1-15, 2019.

[12] Z. Shang, Y. Qiao, L. Duan, and J. Miao, "Stability and bifurcation analysis in a nonlinear harvested predator-prey model with simplified holling type iv functional response," Int. J. Bifurc. Chaos, vol. 30, no. 14, pp. 1-23, 2020.

[13] D. Hu, Y. Li, M. Liu, and Y. Bai, "Stability and Hopf bifurcation for a delayed predatorprey model with stage structure for prey and Ivlev-type functional response," Nonlinear Dyn., vol. 99, no. 4, pp. 3323-3350, 2020.

[14] J. Jia and X. Wei, "On the stability and Hopf bifurcation of a predator-prey model," Adv. Differ. Equations, vol. 2016, no. 1, 2016.

[15] H. El-Owaidy and A. A. Ammar, "Stable oscillations in a predator-prey model with time lag," J. Math. Anal. Appl., vol. 130, no. 1, pp. 191-199, 1988.

[16] P. Holmes and J. Guckenheimer, Nonlinear Oscillations, Dynamical Systems, and Bifurcations of Vector Fields, 1983.
Contribution of Individual Authors to the Creation of a Scientific Article (Ghostwriting Policy)

F. Toledo carried out the modelling, numerical simulation.

P. Cárdenas provided the data and numerical simulation.

C. Escudero was responsible for the final editing of the text in the LaTeX typesetting system and numerical simulation.

Creative Commons Attribution License 4.0 (Attribution 4.0 International, CC BY 4.0)

This article is published under the terms of the Creative Commons Attribution License 4.0 https://creativecommons.org/licenses/by/4.0/deed.en US 